\title{
Energy scheduling in non-residential buildings integrating battery storage and renewable solutions
}

\author{
Lysandros Tziovani, Panayiotis Kolios, Lenos Hadjidemetriou, Elias Kyriakides \\ Department of Electrical \& Computer Engineering, KIOS Research and Innovation Center of Excellence \\ University of Cyprus \\ Nicosia, Cyprus \\ Email: \{ltziov01, pkolios, hadjidemetriou.lenos, elias\}@ucy.ac.cy
}

\begin{abstract}
This paper presents an optimal scheduling for the utilization of the battery energy in order to minimize the cost of the electricity bill in a non-residential building equipped with a photovoltaic-storage system. The battery storage device is connected at the DC-link of the photovoltaic system for enabling a more efficient grid integration through a common central inverter. For the optimal scheduling method, the day-ahead electricity pricing and the next-day forecasted building load and PV generation for each quarter hour are taken into consideration. The problem is formulated as a mixed-integer linear programming model and is solved using commercial solvers. Furthermore, the efficiency curve of the inverter is approximated by a discretized step function and along with the battery efficiency are included in the formulation. Two case studies are carried out using real data and useful results according to the energy flow in the system and the profit are obtained.
\end{abstract}

Keywords-Battery storage, mixed-integer linear programming (MILP), optimization, photovoltaics, scheduling algorithm.

\section{NOMENCLATURE}

\section{A. Indices/Sets}

$T \quad$ Set of optimization time interval.

$I \quad$ Set of discretized parts of the inverter efficiency curve.

$t \quad$ Index for time intervals.

$i \quad$ Index for discretized parts.

\section{B. Parameters}

$c_{\text {grid }}(t) \quad$ Cost of power purchased by the grid in $€ / \mathrm{kWh}$.

$c_{\text {sell }}(t) \quad$ Cost of power sold to the grid in $€ / \mathrm{kWh}$.

$D(t) \quad$ Load demand in $\mathrm{kW}$.

$P_{\mathrm{PV}}(t) \quad$ Power generated by the PV system in $\mathrm{kW}$.

Active power values for discretized inverter

$p_{(i)} \quad$ efficiency in $\mathrm{kW}$.

$e_{(i)} \quad$ Inverter efficiency coefficients in $\%$.

$b_{\mathrm{c}} \quad$ Charging coefficient factor.

$b_{\mathrm{d}} \quad$ Discharging coefficient factor.

$P_{B}^{D \max }$ Maximum discharging power of the battery in $\mathrm{kW}$.

$P_{B}^{C m a x}$ Maximum charging power of the battery in $\mathrm{kW}$.

$S O C_{\text {min }}$ Minimum State of charge of the battery in kWh.

$S O C_{\max }$ Maximum State of charge of the battery in kWh.

IC Initial capacity of the battery in $\mathrm{kWh}$.

The authors are supported by the Research Promotion Foundation (RPF, Cyprus, Project KOINA/SOLAR-ERA.NET/1215/06), by the Ministry of National Infrastructure Energy and Water (Israel) and the SOLARERA.NET (European Union's Seventh Framework Programme).

\section{Variables}

$P_{\text {Abs }}(t) \quad$ Power absorbed from the grid in $\mathrm{kW}$.

$P_{\text {Inj }}(t) \quad$ Power injected to the grid in $\mathrm{kW}$.

$P_{\text {Disch }}(t) \quad$ Discharging power of the battery in $\mathrm{kW}$.

$P_{\mathrm{Ch}}(t) \quad$ Charging power of the battery in $\mathrm{kW}$.

$X_{1}(t) \quad$ Inverter input power at the DC side in $\mathrm{kW}$.

$X_{2}(t) \quad$ Inverter active input power at the AC side in $\mathrm{kW}$.

$Y_{1}(t) \quad$ Inverter active output power at the AC side in $\mathrm{kW}$.

$Y_{2}(t) \quad$ Inverter output power at the DC side in $\mathrm{kW}$.

$S O C(t) \quad$ State of charge of the battery in $\mathrm{kWh}$.

\section{Binary Variables}

$B_{(i)} \begin{aligned} & \text { State of the discretized part of the inverter efficiency } \\ & \text { curve at the DC to AC conversion. }\end{aligned}$
$W_{(i)}$ State of the discretized part of the inverter efficiency
curve at the AC to DC conversion.

\section{INTRODUCTION}

The penetration of the renewable energy sources into the power systems is expected to increase rapidly in the next years due to the European 20-20-20 targets which aim to increase the share of renewable energy in the total energy consumption to $20 \%$ [1]. However, the high penetration of the renewable sources causes several issues to the operation and stability of the power systems due to the intermittent character of these sources [2]. Technologies such as energy storage systems can solve these issues by maintaining the balance of supply and demand, and supporting the stability and the frequency of the grid. To date, batteries are the most well-accepted technology among the alternative energy storage systems which are used in a wide range of applications such as electric vehicles, electric utility energy storage, and storage of electric energy produced by renewable energy sources. Moreover, batteries can reduce the cost of electricity in commercial buildings and residential dwellings by absorbing and storing energy from the grid during off-peak periods and injecting it during high-demand periods when prices are very high [3], [4].

Batteries are used in various systems and through optimization techniques the target is to minimize the operational cost of these systems. In [5], a linear programming method is presented to schedule the micro-grid battery storage system for the next day using the forecasted load, PV generation and spot electricity prices. Similarly, a linear programming method is presented in [6] for the optimal scheduling of a linearized model 
of lithium-ion battery in an industrial micro-grid while taking into account hardware and grid constraints. A Mixed Integer Linear Programming (MILP) formulation of the micro-grid economic scheduling which is composed of the utility grid, distributed generators, storage system, PV generation and both critical and controllable loads is presented in [7], assuming perfect knowledge of the micro-grid state, renewable resources production and future loads. In [8], the methodology in [7] is enhanced in order to cope with inevitable disturbances and forecast errors. A MILP method is proposed in [9] to minimize the residential electricity bill by scheduling the appliances operation over a daily forecast price cycle. In [10], a trade-off between minimizing the payment and minimizing the waiting time for the operation of the household appliances is taken into consideration in an optimal residential energy consumption scheduling framework. References [9] and [10] do not take the storage and photovoltaic integration into consideration. Dang et al. [11] propose an optimal load scheduling algorithm to minimize electricity payment in a smart home with local renewable energy sources and energy storage. In [12], a MILP model and a heuristic technique are presented to optimize the energy production and consumption systems in a smart home with the integration of renewable energy sources, energy storage and electric vehicles in order to minimize the electricity cost of the consumer.

In this paper, a MILP model is presented to schedule the battery energy in order to minimize the cost of the electricity bill in a non-residential building equipped with a photovoltaic and battery system. Taking into account the day-ahead electricity pricing and the next-day forecasted load and PV generation, the algorithm decides when to charge or discharge the battery and when to buy or sell energy for each quarter hour of the next day. In contrast with the residential load, the load of a non-residential building is quite predictable and follows a certain pattern. So, the load scheduling of [9]-[11] is not applicable in this study. Further, the storage system is installed at the DC-link of the photovoltaic system in order to enable the grid integration of both systems through a common inverter. Thus, an increased efficiency can be achieved by the combined system and a decreased initial investment is required. The main contributions are:

a) An optimal solution for a real commercial PV-BatteryInverter system.

b) The inclusion of the inverter exponential efficiency curve which is approximated by a discretized step function.

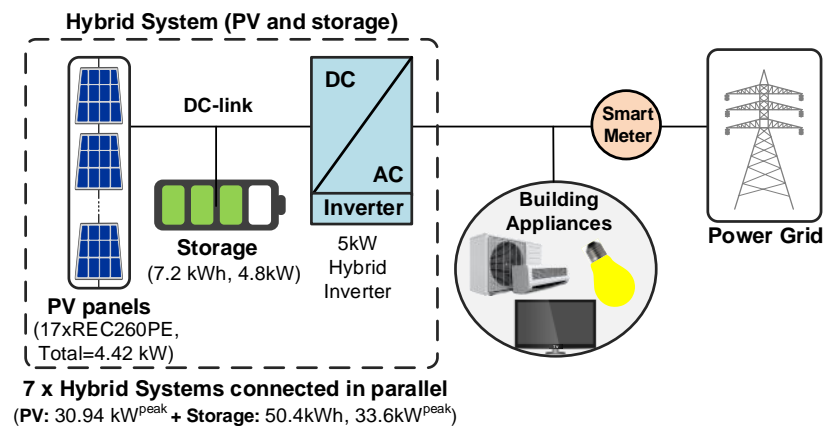

Fig. 1. Physical scheme of the system.
The structure of the rest of the paper is as follows. In Section III, the physical system is described, followed by the problem formulation in Section IV. The simulation results are presented in Section V and the conclusions are given in Section VI.

\section{PHYSICAL SYSTEM}

The physical scheme of the system is illustrated in Figure 1. The whole system consists of a prosumer (PROducer and conSUMER) that is interconnected with the power grid. The prosumer is equipped with loads and a hybrid system which is composed of seven hybrid sub-systems connected in parallel. Each hybrid sub-system includes $17 \mathrm{PV}$ panels connected in series [14], a battery with $7.2 \mathrm{kWh}$ usable capacity range [15] and a $5 \mathrm{kVA}$ hybrid inverter [16]. It is assumed that the inverter delivers only active power during its operation. The total PV capacity of the hybrid system is $30.94 \mathrm{~kW}$, the total usable capacity of the batteries is $50.4 \mathrm{kWh}$ (i.e. $S O C_{\min }=0 \mathrm{kWh}$ and $S O C_{\max }=50.4 \mathrm{kWh}$ ) with $33.6 \mathrm{~kW}$ nominal charging and discharging power, and the total maximum active power of the inverters is $35 \mathrm{~kW}$. However, the maximum charging and discharging instantaneous power of the batteries is reduced to $24.5 \mathrm{~kW}$ instead of $33.6 \mathrm{~kW}$ in the simulations for increasing the lifetime of the batteries. It can be noted that the total maximum power from the PV and the batteries is $55.44 \mathrm{~kW}$ which is higher than the maximum power of the inverters. Consequently, the maximum instantaneous discharging power of the batteries is dependent on the PV production. The total maximum active power of the inverters is selected to be $35 \mathrm{~kW}$ for reducing the capital investment of the total system.

The hybrid inverter allows the bi-directional power flow between the DC and AC side. The produced power from the PV and the exported power from the batteries is consumed on the load and the surplus power is injected into the grid. On the other hand, the batteries are able to store the DC power of the photovoltaics or even to absorb power from the grid for charging. However, the power flow through the inverter is reduced according to the inverter efficiency curve due to the energy losses of the inverter. Figure 2.a shows the exponential efficiency curve of the $5 \mathrm{kVA}$ hybrid inverter which determines how much of the DC power is converted to $\mathrm{AC}$ power and vice versa [16]. Furthermore, the batteries are not ideal but present charging and discharging losses. For the $5 \mathrm{kVA}$ hybrid inverter the maximum efficiency between the PV, battery, and

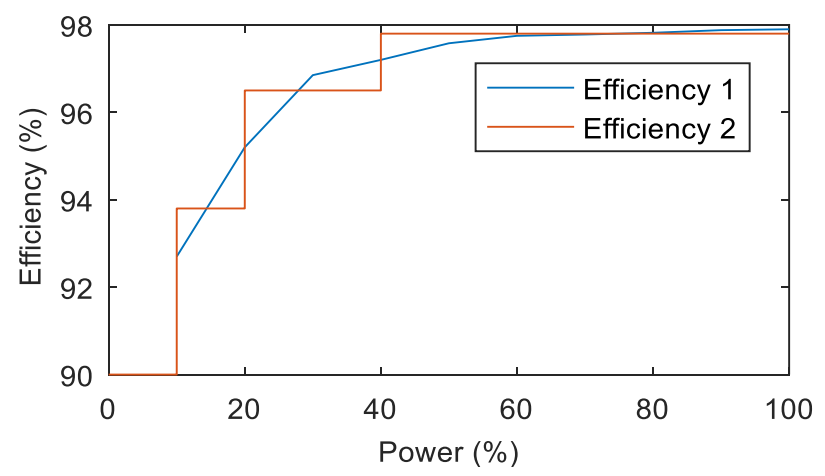

Fig. 2. (a) Efficiency 1: Inverter efficiency curve. (b) Efficiency 2: A discretized approximation of the inverter efficiency. 


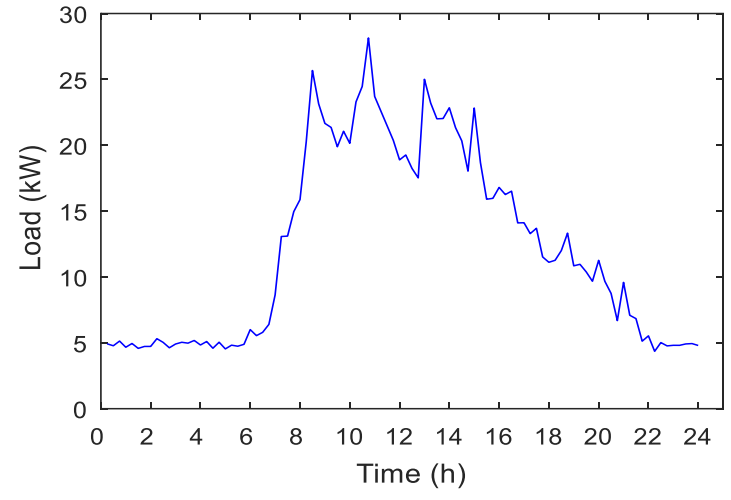

Fig. 3. The load curve of the building for a working day.

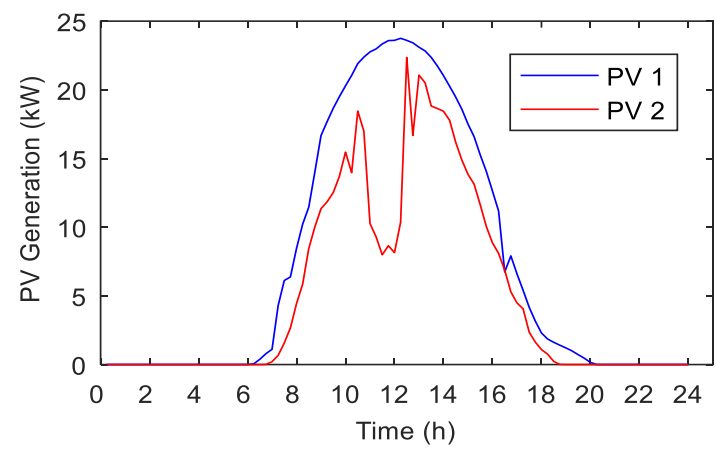

Fig. 4. PV 1: PV production for a sunny day. PV 2: PV production for a shaded day

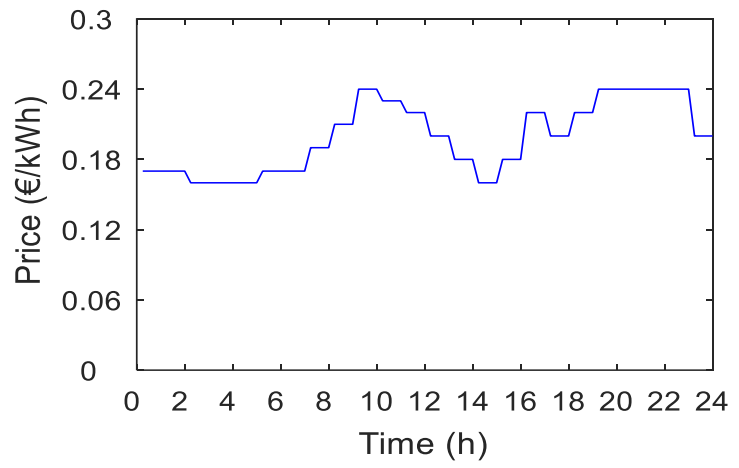

Fig. 5. Day-ahead electricity pricing

the grid is higher than $90 \%$ [16]. In the simulations, the charging and discharging coefficients of the battery are set to $98 \%$.

The load prediction of a single residential load is very challenging due to the load variation which follows the habits of the residents. In contrast, the load of a non-residential building is quite predictable and follows a certain pattern. For example, the load in office buildings is high only during working hours due to the lighting, heating-cooling, and the consumption of office equipment. An example of the load (with a 15 min resolution) of such a non-residential building is presented in Figure 3, according to a measurement campaign performed by the authors.

Similarly, a PV production pattern of two nominal cases is shown in Figure 4, according to real measurements from a PV

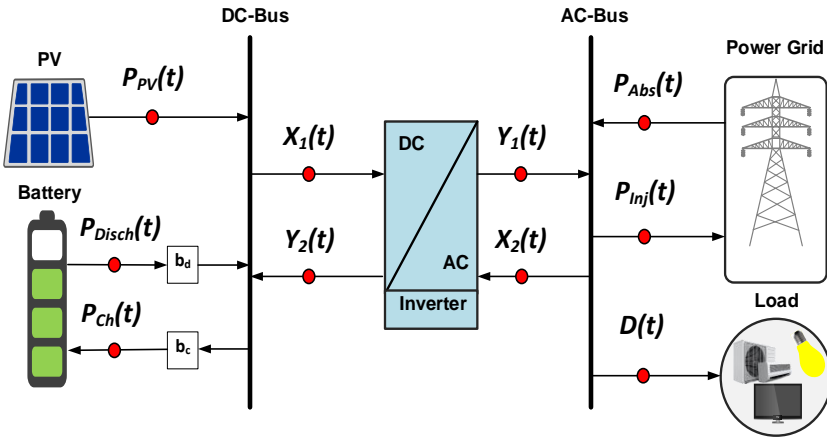

Fig. 6. System model

plant. The PV 1 curve shows the PV production for a typical sunny day and the PV 2 curve shows the PV production for a shaded day. Finally, the day-ahead electricity pricing as it fluctuates over a day is shown in Figure 5 and is assumed to be the same for both electricity purchase and selling. The day-ahead electricity pricing of [13] is used and is normalized according to the electricity price of Cyprus.

\section{PROBLEM FORMULATION}

The equivalent model for formulating the optimization problem is presented in Figure 6 and the system components of each category are represented by their aggregated values using a single component. The model is composed of two buses, the DC and the AC bus. The PV and the battery are connected on the DC bus while the load and the grid are connected on the AC bus. The imported power in the battery is multiplied by the battery coefficient $b_{c}$ and the exported energy from the battery is multiplied by the coefficient $b_{d}$ respectively. Finally, the power flow from the DC side to the AC side and vice versa is multiplied by the inverter efficiency curve. The arrows show the possible energy flow in the system.

Noticeably, the exponential pattern of the inverter efficiency introduces nonlinearities that make computations hard to tackle. For simplifying the problem, the exponential efficiency curve of Figure 2.a can be approximated in a discretized way (as shown in Figure 2.b). Note that the accuracy of the approximation can be adjusted by the numbers of the discretized steps. Using this approximation, a mixed integer linear programming (MILP) technique is formulated hereafter that is solved to optimality and computes the battery scheduling and the power flows that minimize the operating cost while satisfying the demand in a non-residential building.

Specifically, the optimization problem is formulated as an MILP along an arbitrary time horizon $T$. The period of study in this work is set for one day and the time slot is set for 15 minutes. In total, power flow decisions for 96 time intervals are made. Note that in this work deterministic forecasted data values for PV generation, load and day ahead electricity prices are used. Future work will address the uncertainties associated with the aforementioned input data using a stochastic variant of the formulated MILP problem.

\section{A. Objective Function}

The objective of this optimization problem is to minimize the cost of the electricity payment for the whole horizon of study. Consequently, the cost of the imported energy from the grid 
should be minimized and the profit from the energy exported to the grid should be maximized. As such, the objective function can be expressed as follows,

$\min f($ cost $t)=\sum_{t=1}^{T}\left(\frac{c_{\text {grid }}(t)}{4} * P_{\text {Abs }}(t)-\frac{c_{\text {sell }}(t)}{4} * P_{\text {Inj }}(t)\right)$

In (1), $P_{A b s}(t)$ is the absorbed power from the grid in $\mathrm{kW}$; $P_{I n j}(t)$ is the injected power to the grid in $\mathrm{kW} ; c_{\text {grid }}(t)$ is the cost of the absorbed power from the grid in $€ / \mathrm{kWh} ; c_{\text {sell }}(t)$ is the cost of the injected power to the grid in $€ / \mathrm{kWh}$. In the case where the cost of purchase and selling electricity is the same then an infinitesimally small constant can be added to $c_{\text {grid }}(t)$ in order to avoid the simultaneous energy absorption and injection with the grid. Moreover, since we consider time slots of 15 min duration, these cost parameters need to be divided by 4 to account for the cost associated for each fraction of time, as shown in (1).

\section{B. Constraints}

Any feasible solution is subjected to the following constraints:

1) Power Balance on the AC Bus: The power imported in the bus must be the same with the power exported from the bus.

$$
P_{A b s}(t)+Y_{1}(t)=D(t)+P_{I n j}(t)+X_{2}(t)
$$

Variables limits:

$$
P_{A b s}(t), Y_{1}(t), P_{\text {Inj }}(t), X_{2}(t) \geq 0
$$

where $D(t)$ is the load demand in $\mathrm{kW}, X_{2}(t)$ is the inverter active input power at the $\mathrm{AC}$ side in $\mathrm{kW}$, and $Y_{l}(t)$ is the inverter active output power at the AC side in $\mathrm{kW}$.

2) Power Balance on the DC Bus: Similarly with the AC bus, the energy imported in the DC bus must be the same with the energy exported from the DC bus.

$$
P_{P V}(t)+b_{d} * P_{D i s c h}(t)+Y_{2}(t)=b_{C} * P_{C h}(t)+X_{1}(t)
$$

Variables limits:

$$
\begin{gathered}
P_{\text {Disch }}(t), Y_{2}(t), P_{C h}(t), X_{1}(t) \geq 0 \\
P_{D i s c h}(t) \leq P_{B}^{D \max } \\
P_{C h}(t) \leq P_{B}^{C \max }
\end{gathered}
$$

where $P_{D i s c h}(t)$ is the discharging power of the battery in $\mathrm{kW}$, $P_{C h}(t)$ is the charging power of the battery in $\mathrm{kW}, X_{l}(t)$ is the inverter input power at the DC side in $\mathrm{kW}$, and $Y_{2}(t)$ is the inverter output power at the DC side in $\mathrm{kW}, b_{c}$ and $b_{d}$ are the charging and discharging coefficients of the battery, $P_{B}^{D \max }$ and $P_{B}^{C \max }$ are the maximum discharging and charging power of the battery in $\mathrm{kW}$.

3) Battery capacity: The state of charge of the battery in $t$ is measured in $\mathrm{kWh}$ and is expressed as the initial capacity of the battery minus the summation of the discharging power plus the summation of the charging power for all the past and the present time intervals. The charging and discharging power is divided by 4 due to the quarter hour time slots, as previously described.

$$
\operatorname{SOC}(t)=I C-\sum_{j=1}^{t} \frac{1}{4} * P_{D i s c h}(j)+\sum_{j=1}^{t} \frac{1}{4} * P_{C h}(j)
$$

Limits:

$$
\begin{aligned}
& S O C(t) \geq S O C_{\text {min }} \\
& S O C(t) \leq S O C_{\text {max }}
\end{aligned}
$$

where $\operatorname{SOC}(t)$ is the state of charge of the battery in $\mathrm{kWh}, I C$ is the initial capacity of the battery in $\mathrm{kWh}, S O C_{\min }$ and $S O C_{\max }$ are the minimum and maximum states of charge of the battery in $\mathrm{kWh}$. Note that charging and discharging can not occur at the same time due to the input and output losses described in equation (4).

4) Inverter efficiency: As shown in Figure 2.b, an arbitrarily large quantization step could be used to approximate the inverter efficiency. For simplicity, four quantization steps $(I=4)$ are used in this work to approximate the output power of the inverter. Noticeably, the output power of the inverter is the product of the input power and the corresponding efficiency coefficient of the quantized inverter efficiency. The correct coefficient is selected according to the range of the input power.

DC to AC conversion:

$$
Y_{1}(t)=\sum_{i=1}^{I} e_{i} * X_{1}(t) * B_{i}(t)
$$

$\mathrm{AC}$ to $\mathrm{DC}$ conversion:

$$
Y_{2}(t)=\sum_{i=1}^{I} e_{i} * X_{2}(t) * W_{i}(t)
$$

where $e_{i}$ are the efficiency coefficients, $B_{i}(t)$ are the binary variables for the $\mathrm{DC}$ to $\mathrm{AC}$ conversion and $W_{i}(t)$ are the binary variables for the $\mathrm{AC}$ to $\mathrm{DC}$ conversion. For the discretized efficiency shown in Figure 2.b, the efficiency coefficients are selected at $90 \%, 93.8 \%, 96.5 \%$ and $97.8 \%$ of the inverter's efficiency.

5) Limitation of the binary variables: The output option of the inverter in (8) and (9) is satisfied through the restriction of the binary variables. In more detail, the power flow direction through the inverter at each time interval is determined by the binary variable as described below:

$$
\sum_{i=1}^{I} B_{i}(t)+\sum_{i=1}^{I} W_{i}(t)=1
$$

6) Selection of the binary variables: The binary variables are selected according to the range of the inverter input power.

$$
\begin{aligned}
& \sum_{i=2}^{I} p_{i-1} * B_{i}(t)<X_{1}(t) \leq \sum_{i=1}^{I} p_{i} * B_{i}(t) \\
& \sum_{i=2}^{I} p_{i-1} * W_{i}(t)<X_{2}(t) \leq \sum_{i=1}^{I} p_{i} * W_{i}(t)
\end{aligned}
$$

where $p_{i}$ are the active power values for discretized inverter efficiency in $\mathrm{kW}$. For the inverter efficiency in Figure 2.b, the $p_{i}$ coefficients are set to $10 \%, 20 \%, 40 \%$ and $100 \%$ and are multiplied by the maximum power of the inverter. 
(a)

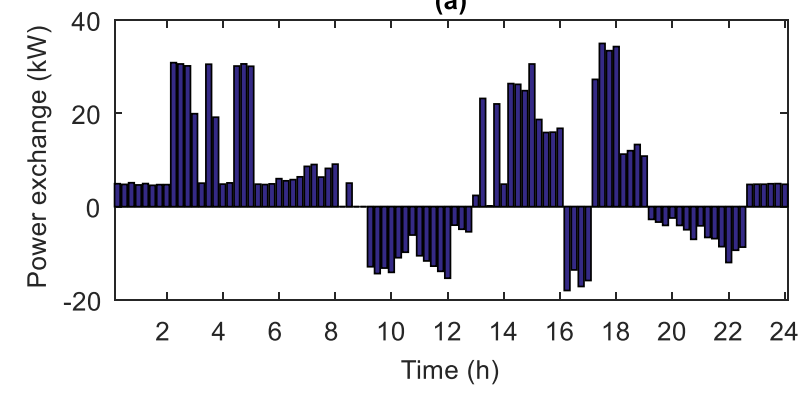

(b)

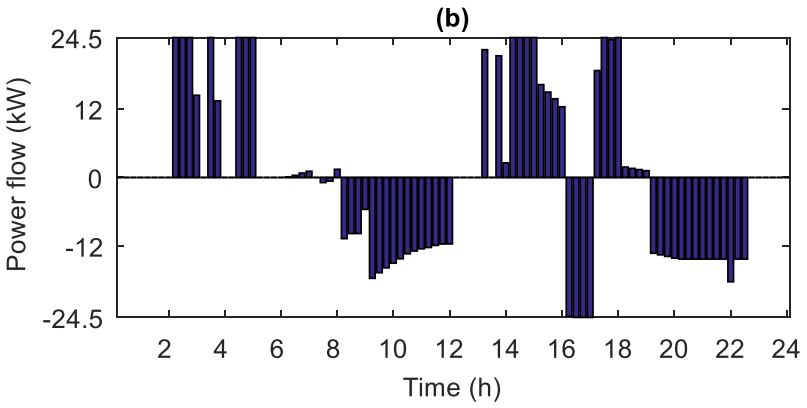

(c)

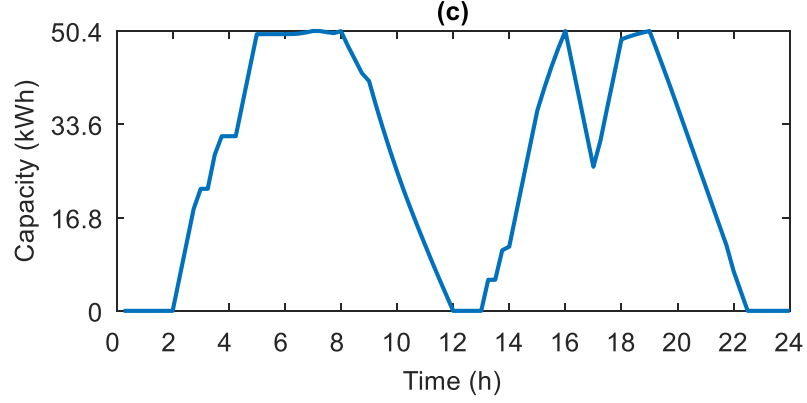

Fig. 7. (a) Purchased/sold power (b) Charge/discharge power of the battery (c) State of charge of the battery.

\section{Simulation Results}

In this section we investigate the performance of the proposed solutions using realistic data for all parameters affecting purchasing, selling, and storing electricity. To do so, the derived MILP formulation described in the previous section is coded in Matlab; Gurobi [17] is used to solve the problem. A standard i5-6200u processor, with 8 GB RAM has been used to solve the problem with an average computation time of approximately 26 s. In what follows, two different case scenarios are investigated to demonstrate the different decisions made on battery scheduling.

\section{A. Case 1}

This case study considers the operation of the system during a sunny day. The load curve, day-ahead electricity pricing, and the PV 1 are used as input data. The optimal battery scheduling which is obtained for every 15 minutes, is shown in Figure 7.b. Positive values denote the charging periods while negative values refer to discharging periods. Similarly with Figure 7.b, Figure 7.a shows the power exchange with the grid. Finally, the state of the charge of the battery is illustrated in Figure 7.c. As expected, during periods of low electricity cost, power is (a)

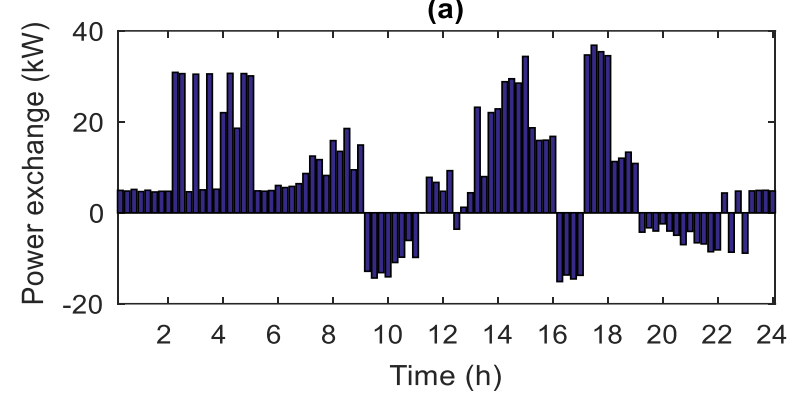

(b)

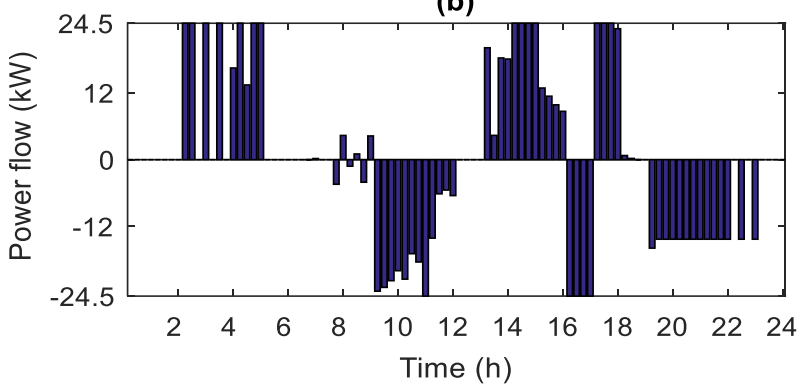

(c)

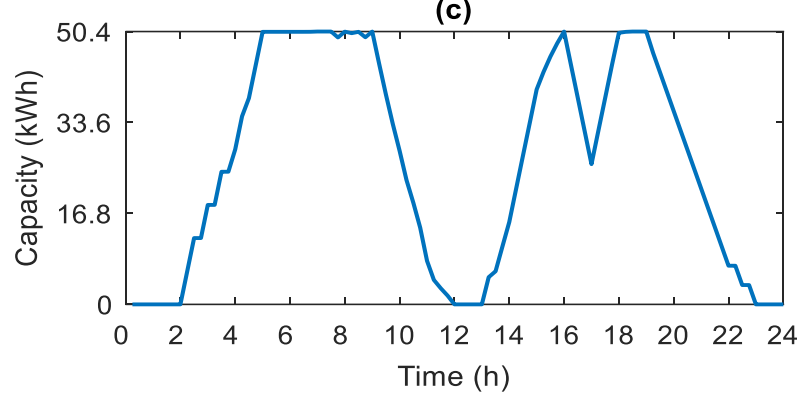

Fig. 8. (a) Purchased/sold power (b) Charge/discharge power of the battery (c) State of charge of the battery.

imported and the battery is charged while during periods of high electricity cost, power is injected to the grid and the battery is discharged. The saturation of the inverter is observed from 9 am to 12 am due to the high PV generation and the discharge of the battery. The PV generation is more than $15 \mathrm{~kW}$ in these time intervals, consequently the battery discharge power is restricted under the $20 \mathrm{~kW}$ due to the $35 \mathrm{~kW}$ limitation of the inverter. So, the discharging of the battery is expanded in longer time period.

Without battery storage, the power flow through the inverter is only at one direction and the generated power from the PV is exported to the grid and is consumed at the load immediately. As shown in TABLE I, the daily electricity cost for the operation of the system without a battery is $24.58 €$.

TABLE I. COST COMPARISON FOR CASE 1

\begin{tabular}{cc}
\hline Configuration & Daily electricity cost $(\boldsymbol{\epsilon})$ \\
\hline Cost with storage & 18.84 \\
\hline Cost without storage & 24.58 \\
\hline
\end{tabular}


On the other hand, in the presence of battery capacity, the proposed optimization technique manages to reduce the daily electricity cost of the system to $18.84 €$ with a daily gain of 5.74 $€$ and a $23.35 \%$ reduction in total cost.

\section{B. Case 2}

The second case examines the operation during a cloudy day and thus, the PV 2 is used as input data instead of PV 1. The optimal battery scheduling is shown in Figure 8.b, the power exchange with the grid is illustrated in Figure 8.a, and Figure 8.c shows the state of charge of the battery. The results are quite similar with the results of Case 1 because the load and the electricity prices remain the same. However, using the shaded PV curve, the discharging power of the battery is increased during the 9 am to 11 am due to the reduction of the PV generation. Consequently, the battery can be fully discharged over shorter time periods especially when the electricity prices are higher. As shown in TABLE II, the daily electricity cost of the system without battery is $36.38 €$ and the cost with the battery is $30.35 €$. It can be noted that the daily electricity costs are increased due to the reduction of the PV generation but the profit of the battery usage is increased to $6.03 €$ and the total cost is reduced by $16.57 \%$.

TABLE II. COST COMPARISON FOR CASE 2

\begin{tabular}{cc}
\hline Configuration & Daily electricity cost (€) \\
\hline Cost with storage & 30.35 \\
\hline Cost without storage & 36.38 \\
\hline
\end{tabular}

\section{CONCLUSIONS}

In this paper, a mixed integer linear programming model is proposed to schedule optimally the battery energy in order to minimize the cost of the electricity bill in a non-residential building with the integration of a photovoltaic and battery system. Furthermore, the exponential efficiency curve of the inverter is approximated by a discretized step function and along with the battery efficiency, these are included in the formulation. As shown in the simulations, the battery imports energy during low electricity prices and exports energy during high electricity prices. Results show that using the optimal scheduling of the battery, the daily cost of electricity is reduced significantly compared to the results without the use of the battery. Hence, the reduced capital investment required by the specific topology with a common inverter for both PV and storage and the profit gained regarding the operation cost of the system and to the scheduling algorithm can improve the sustainability of such system. However, changes in the day-ahead electricity pricing and the forecasted load and PV generation, were shown to affect significantly the scheduling of the battery and the system profit. Forecasting uncertainties can be addressed by reformulating the derived MILP formulation into a stochastic problem which is part of our future work. Also, the capital investment of the PV panels, batteries and the inverters can also be taken into consideration as part of a holistic feasibility study.

\section{REFERENCES}

[1] European Commission, "Europe 2020 strategy," [Online]. Available: https://ec.europa.eu/info/strategy/european-semester/framework/europe2020-strategy en

[2] G. Hug-Glanzmann, "Coordination of intermittent generation with storage, demand control and conventional energy sources," 2010 IREP Symposium Bulk Power System Dynamics and Control - VIII (IREP), Rio de Janeiro, 2010, pp. 1-7.

[3] A. Joseph, and M. Shahidehpour, "Battery storage systems in electric power systems", in Proc. IEEE Power Engineering Society General Meeting, 2006, pp. 8.

[4] N. Garimella, and N. K. C. Nair, "Assessment of battery energy storage systems for small-scale renewable energy integration," in Proc. TENCON 2009 - 2009 IEEE Region 10 Conference, Singapore, 2009, pp. 1-6.

[5] P. Mahat, "A micro-grid battery storage management," in Proc. IEEE Power \& Energy Society General Meeting, Vancouver, 2013, pp. 1-5.

[6] J. Fedjaev, S. A. Amamra, and B. Francois, "Linear programming based optimization tool for day ahead energy management of a lithium-ion battery for an industrial microgrid," in Proc. IEEE International Power Electronics and Motion Control Conference (PEMC), Varna, 2016, pp. 406-411.

[7] A. Parisio, and L. Glielmo, "A mixed integer linear formulation for microgrid economic scheduling," in Proc. IEEE International Conference on Smart Grid Communications, Brussels, 2011, pp. 505-510

[8] A. Parisio, E. Rikos, and L. Glielmo, "A Model Predictive Control Approach to Microgrid Operation Optimization," IEEE Transactions on Control Systems Technology, vol. 22, no. 5, pp. 1813-1827, Sept. 2014.

[9] M. H. Amini, J. Frye, M. D. Ilić, and O. Karabasoglu, "Smart residential energy scheduling utilizing two stage Mixed Integer Linear Programming," in Proc. North American Power Symposium (NAPS), Charlotte, NC, 2015, pp. 1-6.

[10] A. H. Mohsenian-Rad, and A. Leon-Garcia, "Optimal residential load control with price prediction in real-time electricity pricing environments," IEEE Transactions on Smart Grid, vol. 1, no. 2, pp. 120133, Sept. 2010.

[11] T. Dang, and K. Ringland, "Optimal load scheduling for residential renewable energy integration," 2012 IEEE Third International Conference on Smart Grid Communications, Tainan, 2012, pp. 516-521.

[12] F. Y. Melhem, O. Grunder, Z. Hammoudan and N. Moubayed, "Optimization and energy management in smart home considering photovoltaic, wind, and battery storage system with integration of electric vehicles," Canadian Journal of Electrical and Computer Engineering, vol. 40, no. 2, pp. 128-138, Spring 2017.

[13] S. I. Vagropoulos, L. Hadjidemetriou, A. G. Bakirtzis and E. Kyriakides, "Assessment of electric losses minimization in commercial facilities through combined EV charging and PV generation operation," in Proc. MedPower 2014, Athens, 2014, pp. 1-8.

[14] REC260PE Photovoltaics, [Online]. Available: http://www.recgroup.com/sites/default/files/documents/ds_rec_peak_ene rgy series rev_y us.pdf

[15] Fronius Solar Battery 9.0, [Online]. Available: http://www.fronius.com/en/photovoltaics/products/all-products/storageunits/fronius-solar-battery/fronius-solar-battery-9-0

[16] Fronius Symo Hybrid 5.0-3-S, [Online]. Available: http://www.fronius.com/en/photovoltaics/products/home/inverters/froniu s-symo-hybrid/fronius-symo-hybrid-5-0-3-s

[17] Gurobi Optimization, [Online]. Available: http://www.gurobi.com/index 